\title{
Quantum Critical Phase and Lifshitz Transition in an Extended Periodic Anderson Model
}

\author{
M. S. Laad $^{1}$, S. Koley² and A Taraphder ${ }^{2}$ \\ ${ }^{1}$ Institut für Theo. Physik, RWTH Aachen University, Aachen 52056, Germany. \\ 2 Department of Physics and Centre for Theoretical Studies, \\ Indian Institute of Technology, Kharagpur 721302, India.
}

(Dated: October 23, 2018)

\begin{abstract}
We study the quantum phase transition in $f$-electron systems as a quantum Lifshitz transition driven by selective Mott localization in a realistic extended Anderson lattice model. Using DMFT, we find that a quantum critical phase with anomalous $\omega / T$ scaling separates a heavy Landau-Fermi liquid from ordered phase(s). Fermi surface reconstruction occurs via the interplay between, and penetration of the Green function zeros to the poles, leading to violation of Luttinger's theorem in the selective-Mott phase. We show how this naturally leads to scale-invariant responses in transport. Our work is represents a specific (DMFT) realization of the hidden-FL and FL* theories, and holds promise for study of "strange" metal phases in quantum matter.

PACS numbers: PACS numbers: $71.28+\mathrm{d}, 71.30+\mathrm{h}, 72.10-\mathrm{d}$
\end{abstract}

\section{INTRODUCTION}

Magnetic quantum phase transitions (MQPT) from antiferromagnetically ordered metals to heavy landaufermi liquids (HLFL) are believed to occur in a large class of $f$-electron compounds as an appropriate external parameter is tuned ${ }^{1,2}$. Hertz-Moriya-Millis (HMM) ${ }^{3}$ theory "breaks down" for a sub-class of these systems: the whole Fermi surface (FS), or a subset of FS sheets in multi-band cases, is destabilized (becomes "hot"), and an abrupt Fermi surface reconstruction (FSR), together with anomalous scale-invariant power-law responses, accompanies the QPT. These features are also found in near-optimally doped cuprates and favor a quasi-local picture based on an selective Mott transition (SMT) in terms of either a Kondo-RKKY lattice mode $\underline{\underline{4}} \underset{ }{4}$, Hubbard $\underline{6}$ or periodic Anderson lattice mode ${ }^{5,7}$. On a fundamental side, these findings have spawned intense theoretical interest in view of the fact that the specific scale-invariant responses they exhibit are fundamentally at odds with the Hertz-Moriya-Millis (HMM) approaches to quantum criticality: the scale invariant power-laws are only observed as a function of energy, and the responses have very weak momentum (k) dependence.

These findings have spurred intense theoretical activity on various fronts. Slave-boson-Hartree-Fock-plus gauge field fluctuation approaches have extended earlier studies for the $t-J$ model $^{8}$ to the Kondo-RKKY ${ }^{7}$ as well as extended periodic Anderson ${ }^{\underline{5}}$ models. An attractive proposal in this context is the idea of the $\mathrm{FL}^{*}$ metal, which is argued to result from a decoupling of local moments from conduction electrons at the QPT. This $\mathrm{FL}^{*}$ state is exotic, with fractionalised excitations. Within extended-DMFT ${ }^{4}$, such physics (loss of Fermi Liquid coherence) occurs right at the $\mathrm{QCP}$ associated with onset of magnetic order. On the other hand, the $\mathrm{FL}^{*}$ proposal et $a l^{\underline{\underline{T}}}$ is not tied down to this feature; it only requires short-range fluctuating magnetism, but no AF order. A recent proposal of Anderson ${ }^{9}$ argues that the
Kondo lattice must have a "massively non-Fermi liquid" phase, akin to the "hidden-FL" $\underline{10}$, where strong correlations would completely deplete the Landau quasiparticle pole in the fermionic Green's functions in favor of a branch-cut structure (i.e, an incoherent continuum) in the infra-red. Extant dynamical mean-field theoretic (DMFT) works 11 have studied the issue of the FSR in detail within cellular-DMFT. However, the full set of issues raised by the $\mathrm{FL}^{*}$ and the hidden-FL ideas have not been considered (within a fermionic modelling), as far as we are aware.

In the fermionic context, it is also important to inquire whether the features discussed above, and in particular, the $\omega / T$-scaling and FSR, survive additional realistic features: real rare-earth systems of interest have two features neglected in the minimal Anderson lattice model: finite $f$-bandwidth and $\mathbf{k}$-dependent hybridization ${ }^{5}$ : both these features are clearly visible in first-principles LDA one-electron band structure calculations ${ }^{12}$. It is not a priori clear, especially within DMFT approaches, how these additional delocalising features might affect the local critical behavior hitherto considered. For example, it is well-known that one-electron hybridisation is very often a relevant perturbation, and, in DMFT, can cut off infra-red local singular behavior at low energies under certain conditions, for e.g, in the spinless Falicov-Kimball model, a finite interband hybridisation reverts the metallic (non-symmetry broken) state to a correlated LFL. Thus, studying the stability vis-a-vis fragility of the "strange" metal under more realistic conditions is indispensable for understanding the "strange" metal in real systems $1.2,11$.

\section{HAMILTONIAN}

Hence, we investigate a more "realistic" extended-PAM with finite $t_{f f}$, a k-dependent hybridization, $V(\mathbf{k})$, and finite $f-c$ coulomb interaction. All these features are 


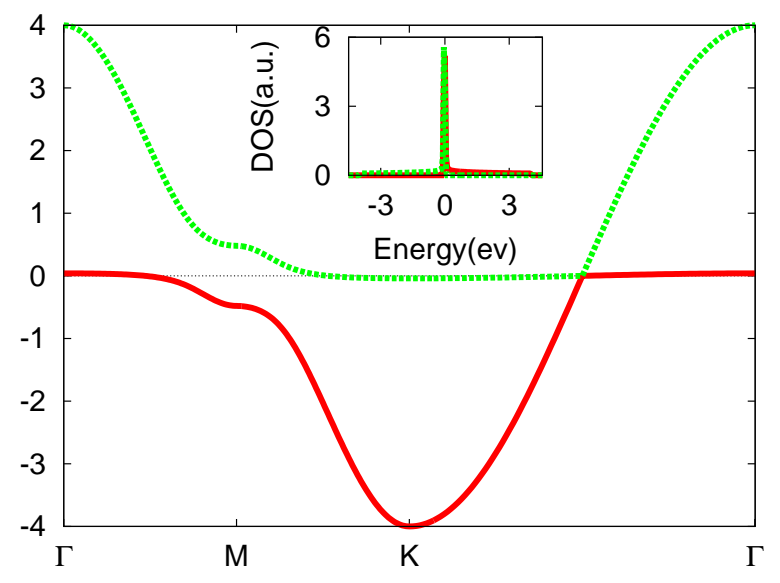

FIG. 1: (Color online) Unperturbed band structure including $d$-wave hybridization for the two-band model in the text.

relevant to a more "realistic" model for the systems of interest: the $f-c$ coulomb interaction, for instance, has long been known as a key factor even for the well-known $\alpha-\gamma$ transition in elemental Cerium ${ }^{13}$. We thus expect that it should have some relevance to real Ce- or $\mathrm{Yb}$ based compounds.

In view of the above, we start with the Hamiltonian $H=$ $H_{0}+H_{1}$ with

$$
\begin{gathered}
H_{0}=-t_{f} \sum_{<i, j>, \sigma} f_{i \sigma}^{\dagger} f_{j \sigma}-t_{c} \sum_{<i, j>, \sigma} c_{i \sigma}^{\dagger} c_{j \sigma} \\
+V_{f c} \sum_{<i, j>, \sigma}\left(f_{i \sigma}^{\dagger} c_{j \sigma}+h . c\right)
\end{gathered}
$$

and the local part is

$$
\begin{gathered}
H_{1}=U_{f f} \sum_{i} n_{i f \uparrow} n_{i f \downarrow}+U_{f c} \sum_{i, \sigma, \sigma^{\prime}} n_{i f \sigma} n_{i c \sigma^{\prime}}+\epsilon_{f} \sum_{i} n_{f i} \\
+V \sum_{i}\left(f_{i \sigma}^{\dagger} c_{i \sigma}+h . c\right)
\end{gathered}
$$

For $U_{f c}=0$, emergence of a heavy LFL within DMFT is well known 18 . Taking the $c$-band centered around $E=0$, we investigate the fate of DMFT Kondo scale in presence of strong, quantum fluctuations of the $f$ occupation, caused by the competition between $V_{f c}$ and incoherence, driven by $U_{f c}$.

\section{DMFT RESULTS AND SELECTIVE-MOTT TRANSITION}

We solve $H$ using the multi-band iterated-perturbation theory (IPT) at arbitrary $T$. Though not numerically exact, it is a fast solver for multi-band cases at arbitrary $T$ and band-filling, recovers known LFL metal for $U_{f c}=0 \underline{18}$, and gives good quantitative accord with oneand two-particle spectral responses for a host of multiorbital strongly correlated systems $\frac{19}{}$. We follow the general DMFT approach ${ }^{20}$, and focus only on symmetryunbroken states hereafter.

The case $\epsilon_{f}=0, V=0$ is analytically soluble in DMFT for a special choice of $V_{f c}^{(c}=\sqrt{t_{f} t_{c}}$. At $V_{f c}^{(c)}$ this becomes the $S=1 / 2$ Falicov-Kimball model (FKM) with $a, b$ fermionic combinations $\underline{21}$ and a rigorous local $\mathrm{U}(1)$ symmetry associated with $\left[n_{i, a}, H\right]=0$ at each site. Within DMFT, $G_{b b}(\omega)$ shows the upper- and lower-Hubbard bands without the renormalised (LFL) lattice Kondo resonance. Concomitantly, $G_{a a}\left(i \omega_{n}\right) \simeq i\left|\omega_{n}\right|^{-(1-\eta)} \operatorname{sgn} \omega_{n}$, and $\alpha=(\delta / \pi)^{2}$ with $\delta=(2 / \pi) \tan ^{-1}\left(\pi \rho_{a}(0) U_{f c} / 2\right)$ is the Anderson orthogonality-catastrophe (OC) exponent, rigorously true for the FKM in DMFT20. Correspondingly, the local excitonic susceptibility, $\chi_{a b}(\omega)=$ $\left\langle a_{i \sigma}^{\dagger} b_{i \sigma} ; b_{i \sigma}^{\dagger} a_{i \sigma}\right\rangle \simeq-i\left|\omega_{n}\right|^{-\left(2 \eta-\eta^{2}\right)}$, is also infra-red singular. This is an analytic demonstration of the intimate link between selective Mott physics and (conformally invariant) local QCP in DMFT. In terms of $f, c$ fermions, the local $G_{f f}$ and $\chi_{f c}(\omega)$ are infra-red singular for a $f_{i-}$ nite $V_{f c}^{(1)}=\sqrt{t_{f} t_{c}}$. A finite $\delta V_{f c}=\left(V_{f c}-V_{f c}^{(c)}\right)$ is known to lead to a finite angular momentum $(l=2)$ LFL for $U_{f c}=022$. Thus, $\delta V_{f_{c}}=0=\epsilon_{f}=0=V$ is a local QCP in the PAM with a finite non-local hybridization, separating two correlated LFL phases: this is a selective Mott metal, and the LFL is destroyed by the OC 10 . Though known for the $\mathrm{FKM}^{4}$, such a local QCP has not been found previously for the PAM with a finite $V_{f c}(\mathbf{k})$ within DMFT. Since $U_{f c}>0$ inhibits lattice Kondo (LFL) coherence in the usual PAM, one expects the local QCP found above to extend into a local non-LFL phase (see below) between critical values $U_{f c}^{(1)}<U_{f c}<U_{f c}^{(2)}$, going over to a Mott insulator for $U_{f c}>U_{f c}^{(2)}$ and a heavy LFL for $U_{f c}<U_{f c}^{(1)}$. Below, we show that such a local QC phase is intimately tied to an orbital-selective Mott localization of the $a$-fermions, and is a manifestation of the fractionalized Fermi liquid $\underline{7}, 15$ within DMFT.

We start with two $(c, f)$ bands in $D=2$, modelled by a simplest nearest-neighbor tight-binding dispersion: $\epsilon_{f, c}(k)=2 t_{f, c}\left(\cos k_{x}+\cos k_{y}\right)$. Motivated by real systems $\frac{1,2}{2}$, we choose $V_{f c}(k)=V_{f c}\left(\cos k_{x}-\cos k_{y}\right)$ to have a $d$-wave form factor, and also keep a small $\epsilon_{f}$ (measured from $E_{F}(=0)$ ). In Fig. 1, we show the unperturbed band structure and local density-of-states (DOS) for selected values of parameters $\left(t_{f}, t_{c}, V_{f c}, V=1.0,0.01,0.24,0\right)$ : this is an input DOS in the DMFT. For concreteness, we choose $U_{f f}=U_{c c}=3.0 \mathrm{eV}$ (intra-orbital Hubbard $U$ ), vary the inter-orbital Hubbard interaction $U_{f c}$, and focus on correlation-induced spectral changes in the DMFT propagators, $G_{a, b}(\omega)$ and self-energies, $\Sigma_{a, b}(\omega)$.

Fig. 2 shows the DMFT spectral functions for the $a, b \stackrel{21}{ }$ bands. Several remarkable features stand out: (i) 
the $a$-band is Mott-split, (ii) the $b$-band DOS clearly shows infra-red singular behavior, with a power-law fall-off in energy upto rather high energy, $G_{b b}(\omega) \simeq$ $c_{1} \theta(\omega)|\omega|^{-(1-\eta)}+c_{2} \theta(-\omega)|\omega|^{-(1-\eta)}$ with $\eta \simeq 2 / 3$ (inset, upper panel, Fig.2), and a pronounced low-energy asymmetry. Correspondingly, at long (imaginary) times, $G_{b b}(\tau) \simeq \tau^{-(2-\eta)}$ instead of $G_{b b}(\tau) \simeq 1 / \tau^{2}$ for any LFL. Now, extending this to finite $T$, Fourier transformating to Matsubara frequencies, followed by an analytic continuation of imaginary frequencies onto the real energy axis, following similar analysis for Luttinger liquids ${ }^{23}$ gives

$$
G_{b b}(\omega, T)=c_{1} T^{-(1-\eta)} F_{0}(\omega / T)
$$

where $F_{0}(\omega / T)$ is a universal scaling function. The onefermion spectral function at finite $T$ thus exhibits $\omega / T$ scaling with an anomalous exponent in the infra-red as a consequence of the Anderson OC, as in the "simpler" toy model above. (iii) concomitantly, $\operatorname{Im} \Sigma_{b}(\omega) \simeq|\omega|^{1-\eta}$, while $\operatorname{Im} \Sigma_{a}(\omega)$ clearly shows a pole at $\omega=0$ characteristic of a Mott insulator, shown in Fig. 4 and (iv) $\operatorname{Re} \Sigma_{b}(\omega)$, shown in upper panel of Fig. 3 clearly reveals vanishing LFL quasiparticle residue, $Z=\left[1-\left(\partial \Sigma_{b} / \partial \omega\right)_{\omega=0}\right]^{-1}=0$, on the FS, implying a divergent effective mass, $m_{b}^{*} / m_{b}=$ $1 / Z$. This singular branch-cut form of $G_{b b}$ is a fundamentally non-perturbative manifestation of selective (Mott) localization, and cannot be obtained in weakcoupling perturbation theory. This OC-induced local critical phase also implies singular hybridization fluctuations: the "excitonic" susceptibility also shows an infrared singularity, $\chi_{a b}(\omega) \simeq \theta(\omega)|\omega|^{-\left(2 \eta-\eta^{2}\right)}$, leading to soft local valence fluctuations $\frac{16}{6}$. This particular aspect might be of interest in connection with the non-LFL behavior of the recently found $\beta-Y_{b A l_{4}}{ }^{17}$

\section{CONNECTION TO HIDDEN-FL AND FL* METALS}

Remarkably, this selective metal is a singular Fermi fluid showing most of the central features of the hidden $\mathrm{FL}^{10}$ and fractionalized $\mathrm{FL}\left(\mathrm{FL}^{*}\right)^{7}$ : (i) implies that $a$-fermion states do not contribute to the reconstructed FS, and Luttinger's theorem does not hold. This is because the pole in $\operatorname{Im} \Sigma_{a}(\omega=0)$ gives a $f_{i}$ nite $\int_{-\infty}^{+\infty}(d \omega / 2 \pi) \Sigma_{a}(\omega)\left(\partial G_{a}(\omega) / \partial \omega\right)=-\operatorname{sign}\left(\epsilon_{f}\right) / 2$, using the effective atomic limit for $G_{a}, \Sigma_{a} \underline{24}$, violating Luttinger's theorem. Though Rosch analysed this case for the Mott insulator within the Hubbard-I approximation, the Mott-localisation of the $a$-band states within DMFT allows us to use the same argument without modification for our metallic case. In reality, the unquenched spin degrees of freedom from the now localised $a$-fermions are now described by an effective spin- $1 / 2$ Heisenberg model at low energy. The superexchange interaction between these spins will now be mediated by the metallic $b$-band carriers, as in an RKKY scenario. An important difference with the usual RKKY case is that the "conduction (a)

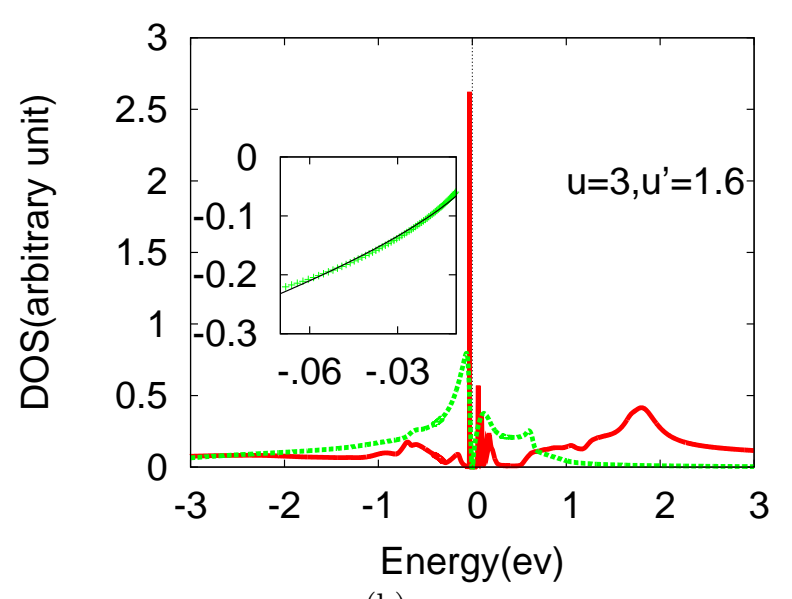

(b)

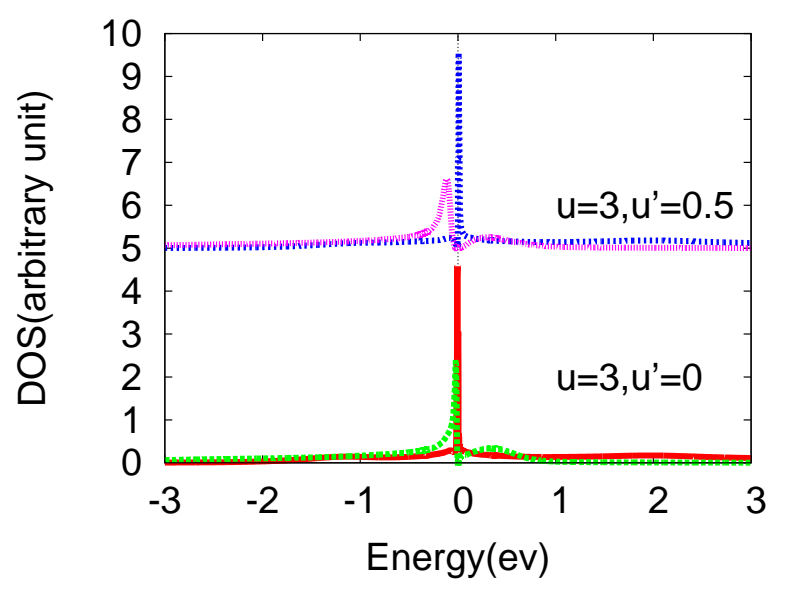

FIG. 2: DMFT DOS for the two band model in the QC metal phase $\left(U_{f c}>U_{f c}^{(1)}\right.$, see text, green and red refer to 'b', 'a' bands) clearly showing power-law fall-off (inset: the black line is the power law fit to the green line, $\operatorname{Im} \Sigma_{b}(\omega)$, in a small range below fermi level) with energy in the infra-red, along with the OS Mott transition (upper panel). Lower panel shows DMFT DOS for the heavy LFL state for $U_{f c}=u^{\prime}=0.0,0.5 \mathrm{eV}$ and $U_{f f}=U_{c c}=u=3.0 \mathrm{eV}$ (red and blue refer to 'a' band; the green and purple refer to ' $b$ ' band).

fermion" sea now corresponds to carriers which are not Landau quasiparticles, thanks to the branch-cut structure of $G_{b b}(\omega)$ found above. This has remarkable consequences when we consider the dynamical spin susceptibility later (see below).

As long as these do not give AF long-range order, our results imply that the spin excitations arising from such a Heisenberg model in the $a$-fermion sector are distinct and asymptotically decoupled from the $b$-fermion states making up the new FS. The $b$-spectral function shows power-law singular behavior of the $(0+1)$-D CFT, $\operatorname{Im} G_{b}(\omega)=\theta(\omega)|\omega|^{-(1-\eta)}$, upto high energy and a pronounced particle-hole (p-h) asymmetry. In hidden-FL 
(a)

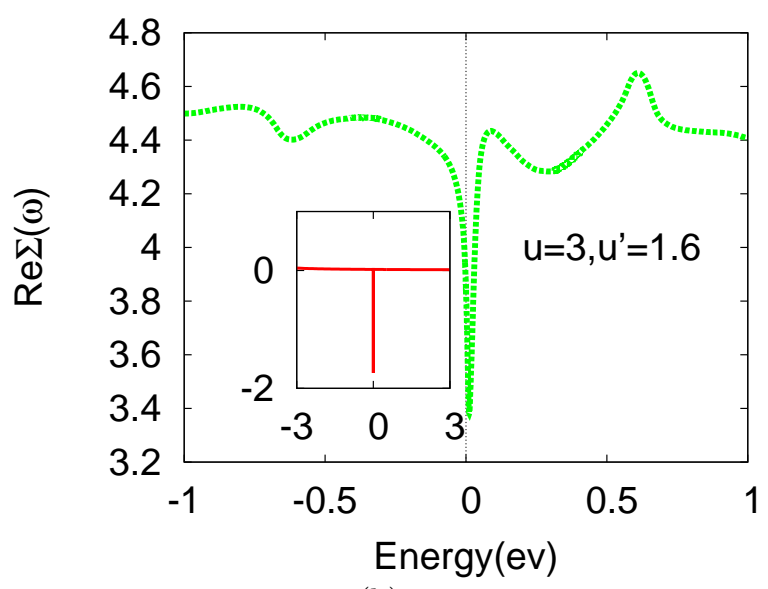

(b)

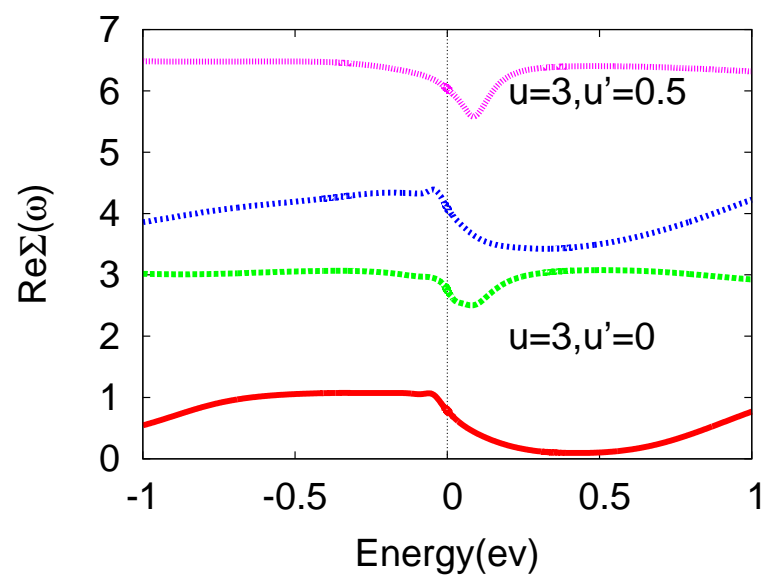

FIG. 3: (Color online) Real part of self-energies for the critical (non-LFL) metal (upper panel) and for the heavy LFL (lower panel) within DMFT. Clear mass divergence and insulating behavior (inset of upper panel, $\mathrm{x}$-axis and $\mathrm{y}$-axis to be multiplied by 0.001 and 1000, respectively) and correlated LFL behavior (lower panel) are manifest. Red (in inset, over a narrow range) and green in upper panel represent the two bands. In the lower panel red and blue stand for band ' $a$ ' while green and purple stand for band ' $b$ ' at two different $u, u^{\prime}$ values.

theory, LFL behavior is destroyed by the OC arising from blocking of recoil processes ${ }^{10}$, while an OSMT is a key requirement for the $\mathrm{FL}^{*}$ state. At least at DMFT level, we see that an OSMT and the OC are inseparable phenomena. Generation of Mott localised $a$-fermion states in the OSMT directly implies strong scattering between localised $(a)$ and "itinerant" $(b)$ fermions: in DMFT, the corresponding impurity model is precisely the X-ray edge problem. The infra-red singular branch-cut form of the DMFT spectra is then a consequence of the lattice "orthogonality catastrophe", very similar to the hiddenFL theory. It also follows that the local QCP is intimately tied to destruction (via $U_{f c}$ ) of the lattice Kondo scale: once the $a$-states are Mott-localized, the $a-b$ hybridization is irrelevant and Kondo screening cannot occur. From (iii), we do find a FS, since $\operatorname{Im} \Sigma_{b}(\omega=0)=0$, but without electron-like quasiparticles $\left(Z=Z_{b}=0\right)$. In contrast to the analytic $S=1 / 2$ FKM argument above, this local critical metal survives a finite range of (see below) $U_{f c}^{(1)}=0.6 \mathrm{eV}<U_{f c}<U_{f c}^{(2)}$ (the "strange" metal goes over to a Mott insulator for $U_{f c}>U_{f c}^{(2)}$, not shown) in the DMFT. Thus, we find a quantum critical phase, again in accord with the hidden FL and $\mathrm{FL}^{*}$ theories. This is a new and novel aspect of our work: earlier DMFT works have focussed on quantum critical point $(s)$ at $T=0$, while we find a $\mathrm{QC}$ phase.

A heavy LFL metal, with correlation-induced Hubbard bands and a low-energy pole in $G_{b b}(\omega)$, with a small but finite $Z$ is already known for $U_{f c}=0 \underline{18}$. A small enough $U_{f c}$ cannot destabilize the lattice (Kondo) LFL coherence scale, so this feature should survive up to a critical $U_{f c}=U_{f c}^{(1)}$. The lower panels in Fig. 2Fig. 4 bear this out, and show low-energy LFL features up to $U_{f c}=0.6 \mathrm{eV}$ instead of OS-Mott-induced nonLFL behavior. Thus, heavy LFL metallic behavior obtains for $0<U_{f c}<0.6 \mathrm{eV}$, while the local "strange" metal is found for $U_{f c}>0.6 \mathrm{eV}$, as promised before. LFL behavior is intimately tied to relevance (in the RG sense) of the hybridization in the PAM, and, when this occurs, (coherent) $a$-spectral weight is pulled down to $E_{F}$, whence no OS-Mott physics can exist in the LFL state. Obviously, the Luttinger volume now encloses both $a$ and $b$-fermions. Since an OS-Mott transition is generically first-order 11 , a discontinuous jump in the FS volume from $V_{F L}=\left[(2 \pi)^{2} / 2 v_{0}\right]\left[\left(n_{a}+n_{b}\right)(\bmod 2)\right]$ to $V_{F L^{*}}=\left[(2 \pi)^{2} / 2 v_{0}\right]\left[n_{b}(\bmod 2)\right]$ rigorously follows across $U_{f c}^{(1)}$ (with $v_{0}$ being unit-cell volume). In the QC phase, the zeros of $G_{a}(\omega)$ cross $\omega=0$ (pole in $\operatorname{Im} \Sigma_{a}(\omega)$ at $\omega=0$ ) for $U_{f c} \geq U_{f c}^{(1)}$, and thus, remarkably, the OSMT with FSR is a correlation-dominated quantum (topolgical) Lifshitz transition (QLT) ${ }^{26}$. In DMFT, the whole $a$-FS sheet disappears at this QLT. On the other hand, in cellular-DMFT, parts of the FS sheet(s) in momentum space would disappear at a momentum-selective Mott transition ${ }^{27}$. The quantum Lifshitz character of the selective-Mott transition is further vindicated by the divergence of the effective mass $\left(m_{b}^{*}=m_{b} / Z_{b}=\infty\right)$ in the QC phase, which implies vanishing quasiparticle stiffness, as expected at a Mottness-induced QLT. We call this a quantum Lifshitz transition, as opposed to a classical one, which occurs, for e.g, in classical frustrated spin systems ${ }^{28}$. Further, it is also very different from a band-Lifshitz transition in a LFL metal: the $a$ band FS sheet vanishes across this quantum transition due to selective Mottness arising from strong electronic correlations. In a usual one-electron picture, this is a simple disappearance of a subset of FS sheet(s) due to band-folding effects, and involves a completely filled oneelectron band, rather than any Mott physics. An important consequence of selective-Mottness, out of scope 
of the "traditional" Lifshitz transition, is that the Luttinger sum rule acquires a new meaning. Recall that, in DMFT, both, $G_{a a}(k, \omega), G_{b b}(k, \omega)$ have the form

$$
G_{\gamma \gamma}(k, \omega)=\frac{1}{\omega-\epsilon_{\gamma}(k)-\Sigma_{\gamma}(\omega)-\frac{V_{\gamma \gamma^{\prime}}^{2}(k)}{\omega-\epsilon_{\gamma^{\prime}}(k)-\Sigma_{\gamma^{\prime}}(\omega)}}
$$

with $\gamma, \gamma^{\prime}=a, b$. In the LFL phase, i.e, as long as $\Sigma_{a, b}(\omega)$ show usual non-singular behavior, $G_{a a, b b}(k, 0)$ can only change sign from positive to negative through an infinity in the G's at a closed FS comprising both $a$ and $b$ fermions. In the selective metal, however, the qualitatively new, singular nature of the DMFT selfenergies introduces qualitatively new aspects. First, since $\operatorname{Im} \Sigma_{b b}(\omega=0)=0$, the $b$-FS sheet is still well-defined, even though LFL quasiparticles have become extinct due to the OC. However, in the selective-Mott phase, the sign change can also occur via a surface of zeros of $G_{a a}(k, \omega)$. This surface of zeros, which is where the zeros of $G_{a a}(k, \omega)$ penetrate what would have been poles in the LFL phase, is entirely distinct from the $b$-FS sheet, and, following Yang et al. ${ }^{30}$, we dub it a Luttinger surface of zeros. The FS in the selective metal encloses the $b$-fermions, while the more generalised Luttinger surface defined above encloses an area given by the total fermion number. Whether this Luttinger surface of zeros can be related to the "ghost Fermi surface" proposed recently ${ }^{31}$ is an interesting question: in our DMFT study, the shortwavelength spin singlet fluctuations (arising from Mottlocalised $a$-fermions as an effective intersite "RKKY" superexchange, see below) will, however, couple to a critical $b$-fermion charge fluctuation spectrum. In Mross et al.'s study, both, the spin-singlet $2 k_{F}$ response and the electrical charge density arise from the same single band. Nonetheless, the formal analogy pointed out here is suggestive, and might facilitate further study of the Luttinger surface in "strange" metals.

Finally, given that the usual Anderson lattice model (ALM) within two-site cluster-DMFT ${ }^{29}$ can be reexpressed as our EPAM in terms of the bonding and antibonding cluster-centered fermionic combinations, similar results, with proper re-interpretation, will follow for the ALM within cluster-DMFT 11 . In this latter case, the self-energy will, of course, explicitly depend on cluster momenta, and, for a two-site cluster, the cluster-DMFT band structure will be modified in a momentum-dependent way at the momentumselective Mott transition. In this case, using the mapping from cellular-DMFT to an effective clustercentered multi-orbital DMFT, the analogous possibility that momentum-selectivity ${ }^{11}$ within cellular-DMFT induces a similar phenomenon of penetration of zeros to poles along certain directions in $\mathbf{k}$-space reveals itself. In underdoped HTSC cuprates, this momentum-selectivity reveals itself as the nodal-antinodal dichotomy and FS pockets in dHvA studies 30 . Thus, whether a DMFT or cluster-DMFT approach should be used must be decided by appeal to $\mathrm{dHvA}$ experiments. If the renormalised band dispersions and FS agree well with LDA, at least in the heavy LFL phase, one can surmise that this $\mathrm{k}$ dependence is then sufficiently weak that DMFT is suffices. If, on the other hand, sizable discrepancy between dHvA results and LDA shows up, one must look at the associated selective-Mott and critical phenomena within cluster-DMFT.

Analytic insight into the non-LFL to LFL "transition" at $U_{f c}^{(1)}$ is obtained by bosonizing the impurity model of DMFT. In the QC metal, the impurity model is that of $b$-fermions scattered by a localized $a$-fermion via $U_{f c}$. This maps exactly onto the X-ray-edge problem, which is bosonizable into a collection of radial (free) bosonic models on a half-line $\mathrm{e}^{32}, 0 \leq r \leq \infty$, i.e, $\left.S=\int_{0}^{\infty} d r\left[\partial_{\tau} \phi_{\alpha}(r)\right)^{2}+\left(\partial_{r} \phi_{\alpha}(r)\right)^{2}\right]$, whence the singularities above can be anaytically derived. Relevance of the hybridization (in the DMFT, this is the LFL phase, where $V_{f c}(k)$ is relevant) necessitates proper treatment of non-adiabatic effects (associated with finite recoil of the $a$-fermion) in the bosonization approach via the lowestorder cluster expansion ${ }^{33}$. Carrying out this procedure changes the singular branch cut structure of $G_{b b}(t)$ above to $G_{b b}(t)=-i \theta(t) \exp \left[-i\left(-\epsilon_{f}+U_{f c} n_{a}\right) t-F_{b}(t)\right]$ with

$F_{b}(t)=\left(U_{f c} / N\right)^{2} \sum_{k, k^{\prime}} n_{k a}\left(1-n_{k^{\prime} a}\right) \frac{1-e^{i \Delta_{a, k k^{\prime}} t}+i \Delta_{a, k k^{\prime}} t}{\Delta_{a, k k^{\prime}}^{2}}$

and $\Delta_{a, k k^{\prime}}==\epsilon_{f}+t_{a, k-k^{\prime}}-t_{a, k}+t_{a, k^{\prime}}$. The $a$-spectral function is now $A_{b}(\omega)=2 \pi Z_{a} \delta(\omega-$ $\left.\epsilon_{a}\right)+A_{\text {inc }}(\omega)$, with a finite $\mathrm{LFL} \mathrm{QP}$ residue, $Z_{b}=$ $\exp \left[-\left(U_{f c} / N\right)^{2} \sum_{k, k^{\prime}} \frac{n_{k a}\left(1-n_{k^{\prime} a}\right)}{\Delta_{a, k k^{\prime}}^{2}}\right]<<1$. The $a$-spectral function also develops a similar (exponentially small) $Z_{a}$ for $U_{f c}<U_{f c}^{(1)}$. For energies $\omega, k_{B} T>\min \left(Z_{a}, Z_{b}\right) E_{F}$, this heavy LFL smoothly crosses over to the QC phase. Interestingly, all these DMFT spectral features, including the exponentially small $Z$ increasing smoothly across $U_{f c}^{(1)}$, closely resemble those found in very recent AdSCFT work ${ }^{14,15}$.

\section{SPIN AND CHARGE FLUCTUATIONS IN THE "STRANGE" METAL}

In this section, we start by observing that anomalous spin and charge correlations have turned out to be defining characteristics of the "strange" metal in cuprates and $f$-electron-based systems. As mentioned in the introduction, several careful studies now show that these have a unique form, namely very anomalous energy dependence, but essentially no k-dependence, that does not fit into the Hertz-Moriya-Millis approach to quantum criticality. In particular,

(i) the dynamical spin susceptibility, $\chi_{\sigma}(\mathbf{q}, \omega, T)$ is essentially independent of $\mathbf{q}$, but shows anomalous powerlaw fall-off in the infrared as a function of $\omega$ : the inter- 
esting finding is that it scales with $\omega / T$, implying no intrinsic energy scale (apart from the temperature itself) in the "strange" metal. This is reminiscent of the marginalFermi liquid idea. However, observation of a fractional exponent (less than unity) in the power-law fall-off is more consistent with the hidden-FL idea.

(ii) similar anomalous behavior is seen in optical conductivity studies in near-optimally doped cuprates as well as $\mathrm{YbRh}_{2} \mathrm{Si}_{2}$.

(i) and (ii) show that both spin and charge correlations are singular in a very anomalous sense in the "strange" metal. To the extent that we are unaware of a calculation where (i) and (ii) follow from analysis of a single model, it is of obvious interest to consider this issue within our approach. We now study these issues.

The fact that the "RKKY" interaction between localized ( $a$-band) moments is now mediated by critical fermionic degrees of freedom, as discussed above, has remarkable consequences for magnetic fluctuations. A localized spin is now "Kondo" coupled to these critical fermions. Alternatively, a local moment experiences a dynamically fluctuating "critical magnetic field" due to the power-law form of $G_{b b}(\omega)$. Explicitly, this coupling is $H^{\prime} \simeq J_{K} \sum_{i} \mathbf{S}_{i, a} \cdot \sigma_{i, b} \simeq J_{K} \sum_{i} \mathbf{S}_{i, a} \cdot \mathbf{h}_{i, a}$, where the "field", $\mathbf{h}_{i, a}=\mathbf{h}_{i, a}(\tau)$ encodes the singular nature of the $b$-fermion spectrum found in DMFT. The effective superexchange generated between neighboring localized moments is thus related to the temporal correlations of this "fluctuating field". This can now be treated at an effective level as a problem of a localized spin system coupled to singular "magnetic field" fluctuations 34 . In our case, the exponent in the singular fluctuations of the "magnetic field" now enter from the singular nature of $G_{b b}(\omega)$ found above. As shown for this simplified problem, the spin-spin correlator now reflects the critical power-law spectrum of the "conduction sea" via coupling to a "critical" bath. In imaginary time, the spin-spin correlator reads $\chi_{s s}^{a}\left(\tau-\tau^{\prime}\right)=J_{K}^{2} \chi_{s s}^{(b)}\left(\tau-\tau^{\prime}\right) \simeq\left(\tau-\tau^{\prime}\right)^{-(2-\mu)}$, where $\chi_{s s}^{(b)}(\tau)$ is the dynamicakl spin susceptibility of the "critical bath". Extracting the functional form of $\mu$ on the exponent of the power-law behavior in $G_{b b}(\omega)$ requires more work. We leave this detail for future work, in keeping with our aim here, which is to qualitatively show how the anomalous magnetic fluctuations are intimately linked to branch cut feature(s) in the one-fermion spectral functions. Extending this to finite $T$, Fourier transforming to energy variables, and analytically continuing the imaginary (Matsubara) frequencies on to the real energy axis yields the dynamical spin susceptibility that can be compared to inelastic neutron scattering results. The dynamical susceptibility is 23

$$
\chi(\mathbf{q}, \omega)=A(\mathbf{q}) T^{-(1-\mu)} F(\omega / T)
$$

, where $F(y)=y^{1-\mu} \sinh (y / 2)\left|\Gamma\left(\frac{\mu}{2}-\frac{i y}{2}\right)\right|^{2}$, i.e, it shows the famous $\omega / T$ scaling. In the heavy LFL state, having a sharp LFL quasiparticle peak in both $G_{a a}, G_{b b}$ now implies that the spin degrees of freedom experience a fluctuating magnetic field as well, but one whose correlations are of the usual LFL type. Namely, the "magnetic field", or the $b$-fermion spin correlator now goes off like $\left(\tau-\tau^{\prime}\right)^{-2}$. Hence, the spin correlation function now shows conventional long-time behavior, $\chi_{s s}^{a}\left(\tau-\tau^{\prime}\right)=$ $J_{K}^{2} \chi_{s s}^{(b)}\left(\tau-\tau^{\prime}\right) \simeq\left(\tau-\tau^{\prime}\right)^{-2}$, characteristic of a LFL, as it must.

We emphasise that a "locally critical" singular magnetic fluctuation spectrum is generated in our case by the local "Kondo" coupling of a local moment (arising from Mott localization of $a$-fermions) to a critical fermionic "bath" (via the infra-red singular $\mathrm{G}_{b b}(\omega)$ ) in the DMFT above. This is physically very different from how a similar effect arises in the Kondo lattice model ${ }^{4}$ : there, it arises as a competition between the local (usual) Kondo effect and the RKKY intersite interactions, precisely at the point where both heavy LFL and magnetic ordering scales are simultaneously suppressed at $T=0$ : it is thus necessarily a quantum criticsl point. In our case, the "strange" metal arises as a phase for a finite range of $U_{f c}^{(1)}=0.6 \mathrm{eV}<U_{f c}<U_{f c}^{(2)}$. In our case, however, there is no need that local-critical magnetic fluctuations be tied to AF order: we only require local coupling of local moments to a critical fermionic bath, and the latter arises in a selective-Mott scenario, as we have found here. Thus, our results are again in accord with the $\mathrm{FL}^{*}$ idea, which does not require the strange metal features to be tied down to onset of magnetic order. While this may be relevant to recent experiments on Co- and Irdoped $\mathrm{YbRh}_{2} \mathrm{Si}_{2}{ }^{35}$, where it is found that the "strange" transition (denoted by $\left.T^{*}(p)\right)$ and the $\mathrm{AF}$ line $\left(T_{N}(p)\right.$ ) do not coincide in general as $T \rightarrow 0$, it is a non-trivial issue in this specific context, possibly involving quantum frustration ${ }^{36}$, and is out of scope of the present work.

Finally, it is also of interest to study the optical conductivity. It has been widely used with success to study correlated systems, and shows unique, ill-understood features in near-optimally doped cuprates and $\mathrm{YbRh}_{2} \mathrm{Si}_{2}$. We show the full optical conductivity in the QC phase in Fig. 5. In DMFT, this involves only the full DMFT propagators, as vertex corrections rigorously drop out due to the locality of the self-energy, or are small enough to be negligible in multi-orbital systems 39 . Physically, the "irrelevance" of vertex corrections in the strange metal can be argued by appeal to DMFT results. In the non-LFL metal, single-electron(hole)-like quasiparticles do not live long enough to effectively interact with each other in the "intermediate" state of the (electron-hole) scattering process; i.e, that the soft multi-particle excitations represented by the incoherent (singular branch-cut) continuum in the DMFT results are scattered before they can recohere into LFL quasiparticles. To the extent that these processes are precisely the ones entering the irreducible vertex in the Bethe-Salpeter equation for the conductivity, their ineffectiveness in the above sense implies that it is a good approximation to ignore vertex corrections. Actually, a similar argument, referred to as 
"holon non-drag" regime in tomographic Luttinger liquid and hidden-FL theories, was previously used for neglecting vertex corrections in the same context ${ }^{38}$. With these methodological clarifications, we find, remarkably, that $\sigma(\omega) \simeq \omega^{-\nu}$ with $\nu \simeq 3 / 4$ up to rather high energy $O(1.0) \mathrm{eV}$, in sharp contrast to the $\omega^{-2}$ form expected for any LFL state. Following Van der Marel et al ${ }^{37}$, we estimate that the transport scattering rate, $\Gamma(\omega) \simeq \omega \cot (\pi \nu / 2)$, linear in $\omega$, while the dynamical effective mass, $m^{*}(\omega) / m \simeq \omega^{-(1-\nu)}$. Such an unusual optical response is one of the benchmarks of the "strange" metal, and is seen in optimally doped cuprates and $\mathrm{YbRh}_{2} \mathrm{Si}_{2}{ }^{37}$.

Thus, our DMFT based selective-Mott scenario yields a consistent description of the "strange" features in both charge and spin fluctuation channels in a single framework.

\section{INSTABILITIES OF THE "STRANGE" METAL}

Finally, the local critical metal we find has a large degeneracy (finite entropy per site) and so can only be a stable state of matter at low but intermediate $T$. It must eventually give way to either a heavy LFL, as described above, or to a multiplicity of (competing) ordered states. Irrelevance of one-electron mixing in our case is reminiscent of coupled $D=1$ Luttinger liquids ${ }^{38}$, where irrelevance of one-particle interchain hopping favors two-particle inter-chain coherence and ordered states. In our case, in analogy with the $D=1$ case, irrelevance of $V_{f c}(k)$ in the QC metal favors intersite and inter-orbital two-particle coherence via $H_{r e s} \simeq\left(V_{f c}^{2} / U_{f c}\right) \sum_{<i, j>, \sigma, \sigma^{\prime}}\left(a_{i \sigma}^{\dagger} b_{j \sigma} b_{j \sigma^{\prime}}^{\dagger} a_{i \sigma^{\prime}}+h . c\right)$. Within DMFT, an exact (Hartree-Fock) decoupling of $H_{\text {res }}$ gives two mutually competing $\mathrm{p}-\mathrm{h}$ and $\mathrm{p}-\mathrm{p}$ order parameters, $\Delta_{a b}^{(1)}=\left\langle a_{i \sigma}^{\dagger} b_{j \sigma}\right\rangle$ and $\Delta_{a b}^{(2)}=\left\langle a_{i \sigma}^{\dagger} b_{j,-\sigma}^{\dagger}\right\rangle$, describing p-h and p-p order parameters, $\Delta_{a b}^{(1)}=\left\langle a_{i \sigma}^{\dagger} b_{j \sigma}\right\rangle$ and $\Delta_{a b}^{(2)}=\left\langle a_{i \sigma}^{\dagger} b_{j,-\sigma}^{\dagger}\right\rangle$, describing unconventional excitonic and superconductive orders (with $V_{f c}(k) \simeq\left(\cos k_{x^{-}}\right.$ $\left.\cos k_{y}\right)$, these are $d$-wave ordered states at $\left.T=0\right)$. In general, the type and symmetry of the ordered states resulting from the QC metal will thus be dictated by the specific $\mathbf{k}$-dependent form-factor of the residual interactions. This is radically different from the normal state(s) and instabilities of a weakly correlated LFL, which crucially depend on band-nesting and/or saddle point features in the LDA band structures, and cannot, by construction, lead to power-law singularities in $D>1$.

\section{CONCLUSION}

In conclusion, we have shown that a quantum critical metallic phase, originating from selective-Mott transition via the OC, is an intrinsic property of the EPAM, at least (a)

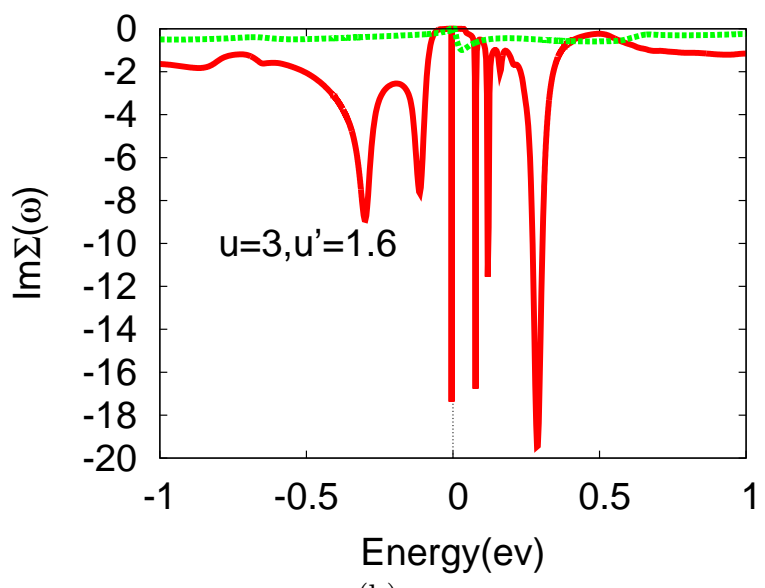

(b)

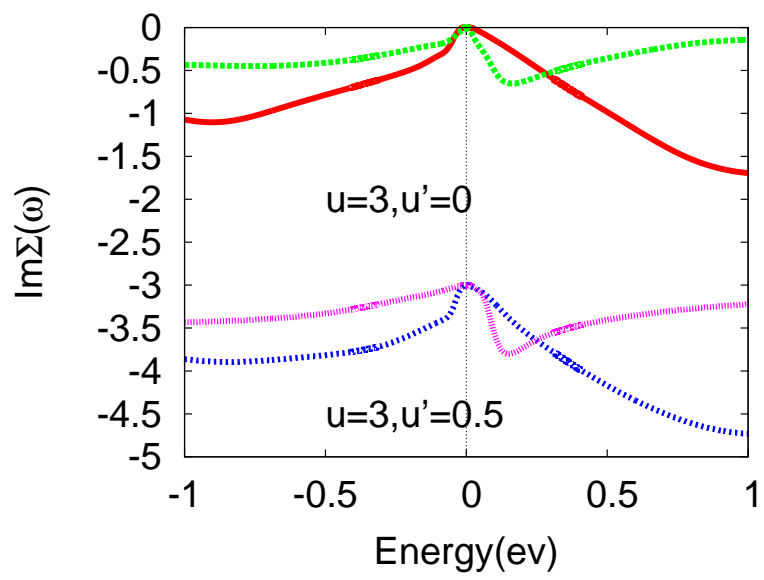

FIG. 4: (Color online) Imaginary parts of the self-energies for the critical metal (upper panel) and the heavy LFL (lower panel) within DMFT. Clear non-LFL behavior (green) and selective Mott (red) behavior is seen (upper panel) and lowenergy heavy LFL behavior (lower panel) is visible $\left(u, u^{\prime}\right.$ as shown in figure, rest of the parameters same as in fig.1).

within DMFT. Both, the famed $\omega / T$ singularities in response functions and the FS reconstruction, now understood as a quantum Lifshitz transition, naturally fall out as consequences of selective-Mott physics. This critical metallic phase is found to either revert back to a heavy LFL as hybridization is tuned to relevance, or argued to give way to (unconventional) competing orders via direct two-particle instabilities. Our findings are in close accord with the hidden-FL and $\mathrm{FL}^{*}$ theories, and hold promise for understanding the "strange" metal phases in quantum matter. 


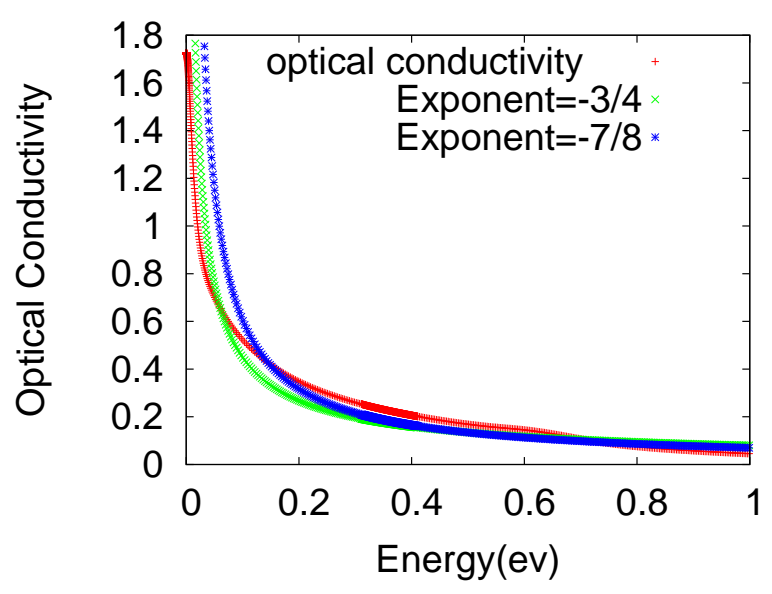

FIG. 5: (Color online) Optical conductivity in the critical metal ( $u=3, u^{\prime}=1.6$, other parameters same as fig.1), clearly showing $\omega^{-\nu}$ behavior with $\nu \simeq 3 / 4$ at low energy, in strong contrast to a smeared lorentzian expected for any heavy LFL state.

1 C. Pfleiderer et al., Nature 414, 427, (2001).

2 P. Gegenwart et al., Nature Physics 186 (2008).

3 J.A. Hertz, Phys. Rev. B 14, 1165 (1976); T. Moriya: Spin Fluctuations in Itinerant Electron Magnetism, Springer Series in Solid State Sciences 56 (Springer, Berlin, 1985).

${ }^{4}$ Q. Si et al., Nature 413, 804, (2001).

5 C. Pepin, Phys. Rev. Lett. 98, 206401 (2007).

${ }^{6}$ K. Haule and G. Kotliar, Phys. Rev. B 76, 104509 (2007).

7 T. Senthil et al., Phys. Rev. Lett. 90, 216403 (2003);

8 P. A. Lee, et al., Rev. Mod. Phys. 78, 17 (2006).

9 P. W. Anderson, Phys. Rev. Lett. 104, 176403 (2010).

10 P.W. Anderson, Nature Phys. 2, 626 (2006)

11 L de Leo et al., Phys. Rev. Lett. 101, 256404 (2008).

12 S. Friedemann et al., Phys. Rev. B, 82, 035103 (2010).

13 J. W. Allen and R.M. Martin, Phys. Rev. Lett. 49, 1106 (1982).

14 S. Sachdev, Phys. Rev. Lett. 105, 151602 (2010).

15 M. Cubrovic et al., Science 325, 439, (2009).

16 S. Watanabe et al., Phys. Rev. Lett. 100, 236401 (2008).

17 S Nakatsuji et al., Nature Physics 4, 603 (2008).

18 N.S. Vidhyadhiraja et al., Euro Phys. Lett. 49, 459 (2000).

19 M.S. Laad et al., Phys. Rev. Lett. 91, 156402 (2003).

20 A. Georges et al., Rev. Mod. Phys. 68, 13 (1996).

21 M.S. Laad et al., Europhys. Lett. 88, 20008 (2009).

22 P. Ghaemi et al., Phys. Rev. B 75, 144412 (2007); ibid Phys. Rev. B 77, 245108 (2008).
23 S. Sachdev et al., Phys. Rev. B 50, 258 (1994).

24 A. Rosch, Eur. Phys. Journ. B 59, 495 (2007).

25 M. Jarrell et al., Phys. Rev. Lett 70, 1670 (1993).

26 M. Imada et al., J. Phys.: Condens. Matter 22, 164206 (2010).

27 M. Civelli et al., Phys. Rev. Lett. 95, 106402 (2005).

28 P. Chaikin and T. C. Lubensky, "Principles of Condensed Matter Physics", Cambridge University Press (1995).

29 A. Liebsch et al., Phys. Rev. B 78, 165123 (2008).

${ }^{30}$ K-Y Yang et al., Phys. Rev. B 73, 174501 (2006).

31 D.F. Mross and T. Senthil, Phys. Rev. B 84, 041102 (2011).

32 K. D. Schotte et al., Phys. Rev. 182, 479 (1969).

33 E. Müller-Hartmann et al., Phys. Rev. B 3, 1102 (1971).

34 A.M. Sengupta, Phys. Rev. B 61, 4041 (2000).

35 S. Friedemann et al., Nature Phys. 5, 465 (2009).

36 J. Custers et al., Phys. Rev. Lett. 104, 186402 (2010).

37 D van der Marel et al., Nature 425, 271 (2003) S. Kimura et al., Phys. Rev. B 74, 132408 (2006)

38 "The Theory of Superconductivity in the High-Tc Cuprate Superconductors", P.W. Anderson, Princeton Series in Physics (1997).

39 J. Tomczak and S. Biermann, Phys. Rev. B 80, 085117 (2009). 